\title{
Quantifying organic carbon burial during the Toarcian Oceanic Anoxic Event
}

\author{
BENJAMIN C GILL', JEREMY D OWENS ${ }^{2}$
}

Dept. of Geosciences, Virginia Tech, Blacksburg, VA USA

2 Dept. of Earth, Ocean and Atmospheric Science,

Tallahassee, FL USA

One of the best-studied global environmental perturbations to the ocean-atmosphere system during the Mesozoic is the Toarcian Oceanic Anoxic Event or T-OAE ( 183 Ma) of the Jurassic Period. The environmental changes during this event resulted in the burial of significant accumulations of organic carbon (OC) and a 3 to $4 \%$ positive carbon isotope excursion in the sedimentary record. Importantly, understanding the amount of $\mathrm{OC}$ buried during this event is critical to constraining its consequences on the global carbon and oxygen budgets and the biogeochemical feedbacks that occurred during this time interval.

Here we will present quantitative global estimates for OC burial during the T-OAE using two methods: (1) calculated mass accumulation (MA) of OC derived from a database of over 100 Toarcian stratigraphic successions and (2) numerical carbon cycle modelling using the Toarcian carbon isotope record. The calculated database estimates for global OC burial, give preliminary estimates of $\sim 10,000$ gigatons of OC. Numerical modelling provides a large range of nonunique estimates for global $\mathrm{OC}$ burial during the event and range between 30,000 to 80,000 gigatons of OC. While the OC MA documented at known sites accounts for significant portion of the required OC buried during the OAE there is significant discrepancies between the two methods. The likely reasons for these differences are the preserved sedimentary record includes mostly marginal marine and epicontinental settings and significant geographic areas are underrepresented in the database: the extrapolated database accounts for $\sim 30 \%$ of the ocean floor. These discrepancies imply that there was significant OC burial associated with this event in sedimentary sequences in unstudied regions or that was subsequently lost through weathering and erosion. 